\title{
Discussion on the Application of Artificial Intelligence in Computer Network Technology
}

\author{
Ganghua Yao ${ }^{1, a}$ \\ ${ }^{1}$ Institute of Technology,East China Jiaotong University, 330100 \\ ayaoganghuajx@126.com
}

Keywords: application; network management; artificial intelligence; computer network technology

\begin{abstract}
Now with the rapid development of science and technology, the computer network technology has been upgraded constantly and the computer technology has been applied more and more extensively, which bring a lot of convenience to people's life. Therefore, there are more and more people on the Internet. The applications of network technology have exerted profound influence on the artificial intelligence. Moreover, the continuous updating and development of artificial intelligence has also led to the continuous innovation and improvement of computer technology. At present, each country in the world has increased the investment and has paid more attention to the artificial intelligence. Through the analysis of the current development situation and the existing applications of artificial intelligence, this paper expounds the role of artificial intelligence during the continuous development of computer network technology.
\end{abstract}

The rapid development of science and technology has brought great convenience to people's life and promoted the progress of computer technology. Nowadays, our needs for change in computer technology are also growing. For example, the great convenience brought by the mobile payment and Smart City has become difficult to meet the needs of people's work and life just in terms of data or simple arithmetic. Therefore, the development of intelligent products is very popular. From those intellectual products of smart mobile phone and smart TV, we can find that people begin to rely on the intelligent technology. The application of artificial intelligence will become more and more extensive during the development of computer network technology.

\section{Related Concepts of Artificial Intelligence}

Artificial intelligence mainly refers to the use of computing technology in order to achieve human thinking and behavior and achieve the intelligent computer technology through the simulation of computer technology. In order to realize the simulation of artificial intelligence, we need to start from many aspects. Firstly, we need to realize the simulation of thinking mode, so people will obtain a better experience in the use through the simulation technology of computer; secondly, we need to realize the simulation of sensory behavior in order to gain more convenient use. In the development of artificial intelligence, the development mode is not single. The development of artificial intelligence not only needs close connection and mutual influence with other subjects, but also needs the mutual cooperation between computer and information technology as well as the influence by the development of computer and information technology. The artificial intelligence is characterized by the integration of different disciplines, different technologies, different resources and data in order to realize the embodiment of intelligence, mutual transmission of information, and sharing and exchange of resources.

\section{Characteristics of Artificial Intelligence}

The emergence of artificial intelligence is just to meet the people's continuous improvement of the quality of work and life. For example, now the development of computer technology is constantly improving and the speed of data processing is very fast. The artificial intelligence will be able to make it more humane, so that it will be more applicable to human life and work. Nowadays, 
many companies has started to develop artificial intelligence step by step and realize the development of artificial intelligence.

Firstly, the artificial intelligence can provide a good solution to this problem in terms of some affirmative answers. Due to the artificial intelligence, we can conduct the calculation even when we has not gained the concrete model. The artificial intelligence can solve problems rapidly and accurately, so the network management can handle some uncertain difficult miscellaneous diseases effectively. Therefore, the network management can be carried out smoothly and the network can be reorganized well.

Secondly, the development of artificial intelligence also needs the continuous learning and expansion of new knowledge. The realization of artificial intelligence reflects that people will be able to use some lower level data to gain the corresponding data through the logical calculation of artificial intelligence. Therefore, we need the better practical application about network management. We need to apply the higher level data and obtain the faster and more intelligent manifestation.

Thirdly, the artificial intelligence will be able to deal with some more complex issues easily with the less resource consumption. We may realize the calculation of complicated arithmetic through the high speed operation effect of artificial intelligence. We may input some fuzzy data and then obtain the best through the internal complex and high speed operation of artificial intelligence, which can save time effectively and achieve a great advantage of improvement of running speed.

\section{Application of Artificial Intelligence in Computer Network Technology}

Firstly, the system evaluation and network management of computer network technology. The realization of intelligent computer network management not only needs the artificial intelligence but also needs the infiltration of telecommunication technology. It should be based on the combination of the two. The artificial intelligence can be applied to conduct network security management. In addition, the problem solving advantage of artificial intelligence itself and some expert knowledge base built through artificial intelligence also can be applied to ensure the integrated management of network. The computer network is characterized by the specific transient and dynamic character, which increases the difficulty of management. We may apply the existing expert system to the artificial intelligence, so the experience and skills will be summarized. Next, we may add the experience into the system and achieve the solution to the problem in the field. Such realization may conduct evaluation and management of the system through the expert system in network management, so as to improve the performance and level of network management.

Secondly, the artificial intelligence agent technology. The software entity of artificial intelligence agent technology is also called the intelligent agent technology. As a software entity, the communication agent is responsible for explaining the inference engine. In the database and knowledge base in the field of knowledge, each agent area library will complete related tasks effectively based on the data processing and communication of new information. Generally speaking, the artificial intelligence agent technology will be able to search information automatically based on the definition of users and transfer it to a specified location so as to provide the intelligent and humanized service for users. For example, when a user is searching information with the computer, the artificial intelligence agent technology will be able to analyze and handle the information and transfer the effective information to the user, so as to save the searching time. In addition, the artificial intelligence agent technology has been widely used in people's daily life, such as the sending and receiving of messages, meeting arrangement, scheduling program, and online shopping, which can provide the quality service for people. At the same time, the technology has some learning and autonomy, since it can help the computer to complete the tasks assigned by the user, which will promote the orderly development of computer technology and network technology.

Thirdly, network security management. In terms of the application of artificial intelligence to 
network security management, we may carry out the discussion and analysis from three aspects. In the first place, the intrusion detection. As for the security management of computer network, the intrusion detection is not only an important part of network security, but also a core part of firewall technology, since it can effectively guarantee the security and reliability of the network. Next, the intelligent firewall. Compared to other defense systems, the intelligent firewall system has been provided with obvious differences, since it can analyze and identify the information and conduct data processing with the intelligent recognition technology, such as decision making, probability, statistics, and storage, which will be able to reduce the amount of computation, timely intercept and restrict the access to invalid and harmful information, and ensure the security of data information. At last, the intelligent anti spam. Adopting mainly the artificial intelligence technology, the intelligent anti spam system can effectively monitor the user's mailbox and automatically scan and identify spam, so as to ensure the security of user information. When the mail is put into the mailbox, the system will send the spam classification information to the user and then the user can conveniently deal with spam in a timely manner, which ensures the security of email system.

\section{Summary}

Along with the continuous development and update of science and technology, the artificial intelligence has achieved great progress continuously and people's work and life are more and more dependent on computer network technology. It is vitally important to realize the embodiment of artificial intelligence in the computer network technology. At the same time, the artificial intelligence has played a considerable role in network management. The artificial intelligence will play a more and more big role when it is applied to computer network technology now and in the future. The artificial intelligence in computer network possesses a strong application prospect. With people's continuous research and exploration of artificial intelligence technology, the artificial intelligence will be applied to more and more extensive areas in the future.

\section{References}

[1] Chuanlin Wei. Discussion on the Application of Artificial Intelligence in Computer Network Technology [J]. Computer Knowledge and Technology, 2015,29:151-152.

[2] Cheng He. Discussion on the Application of Artificial Intelligence in Computer Network Technology [J]. Information \& Communication, 2016,03:180-181.

[3] Ming Mao. Research on the Application of Artificial Intelligence in Computer Network Technology [J]. Electronic Technology and Software Engineering, 2016,09:255-256.

[4] Yifei Peng. The Application of Artificial Intelligence in Computer Network Technology [J]. Scientific Consultation (Technology and Management),2016,06:38.

[5] Shiping Tan. Analysis on the Application of Artificial Intelligence in Computer Network Technology [J]. Silicon Valley, 2013,18:11+4. 